\title{
CONSENSUS
STATEMENT
}

\section{Consensus terminology for preclinical phases of psoriatic arthritis for use in research studies: results from a Delphi consensus study}

\author{
Lourdes M. Perez-Chada ${ }^{1,12}$, Rebecca H. Haberman (10), 212, Vinod Chandran ${ }^{3,4}$, \\ Cheryl F. Rosen ${ }^{5}$, Christopher Ritchlin ${ }^{6}$, Lihi Eder ${ }^{7}$, Philip Mease ${ }^{8,9}$, Soumya Reddy ${ }^{2}$, \\ Alexis Ogdie ${ }^{10}$, Joseph F. Merola ${ }^{1,11,13 凶}$ and Jose U. Scher ${ }^{2,13 凶}$
}

Abstract | The concept of psoriatic arthritis ( $\mathrm{Ps} A$ ) prevention is gaining increased interest owing to the physical limitation, poor quality of life and low remission rates that are achieved with current therapies for PsA. The psoriasis-to-PsA transition offers a unique opportunity to identify individuals at increased risk of developing PsA and to implement preventive strategies. However, identifying individuals at increased risk of developing PsA is challenging as there is no consensus on how this population should be defined. This Consensus Statement puts forward recommended terminology from the Psoriasis and Psoriatic Arthritis Clinics Multicenter Advancement Network (PPACMAN) for defining specific subgroups of individuals during the preclinical and early clinical phases of PsA to be used in research studies. Following a three-round Delphi process, consensus was reached for three terms and definitions: 'increased risk for PsA', 'psoriasis with asymptomatic synovio-entheseal imaging abnormalities' and 'psoriasis with musculoskeletal symptoms not explained by other diagnosis'. These terms and their definitions will enable improved identification and standardization of study populations in clinical research. In the future, as increasing evidence emerges regarding the molecular and clinical features of the psoriasis-to-PsA continuum, these terms and definitions will be further refined and updated.

Psoriatic arthritis (PsA) is a chronic, immune-mediated inflammatory disease, characterized by both skin and joint involvement. Synovio-entheseal involvement is present in up to $30 \%$ of those with psoriasis ${ }^{1,2}$, and individuals with psoriasis progress to PsA at a rate of up to $3 \%$ per year ${ }^{3}$. PsA can lead to joint erosions and deformities ${ }^{4}$, as well as to decreased quality of life $\mathrm{e}^{5}$, high levels of psychosocial stress ${ }^{6}$ and increased rates of comorbidities, unemployment, absenteeism and productivity loss ${ }^{7}$. Despite this burden, PsA remains both underdiagnosed and undertreated, even within dermatology practices ${ }^{8,9}$. The current challenges in diagnosing and treating PsA produce a considerable gap in the care of patients with psoriatic disease, given that a delay in treatment of as little as 6 months can lead to worse disease outcomes ${ }^{10,11}$.

Highly effective treatment strategies are a major measures. These last have become a focus in the field of PsA research and will be aimed at defining, predicting and, ultimately, preventing synovio-entheseal inflammation. To this end, it will be necessary to identify individuals at increased risk of developing PsA and to characterize clinical and molecular features that are specific to preclinical stages of disease. Distinguishing these high-risk individuals will help to shape the development, design and implementation of PsA prevention trials. In addition, it will enable improved screening, earlier diagnosis, timely treatment initiation and, eventually, should improve overall disease outcomes.

The Psoriasis and Psoriatic Arthritis Clinics Multicenter Advancement Network (PPACMAN) is an international non-profit organization that aims to "optimize the clinical care of patients with psoriatic disease through multidisciplinary collaboration, education and innovative research"12. Within PPACMAN, the Preventing Arthritis in a Multicenter Psoriasis At-Risk Population (PAMPA) study group was established to 
understand the clinical, genetic, environmental and immune events that occur during the natural history of the psoriasis-to-PsA transition ${ }^{13}$. To facilitate research focused on the preclinical and early clinical phases of PsA, the PAMPA study group conducted a consensus-building exercise to agree on common terminology related to the preclinical phases of PsA for use in clinical trials and translational research. The development of standardized nomenclature and common definitions to be used exclusively for research in this area should help in the recruitment of well-defined, homogeneous cohorts of patients and enable comparison across future trials and experimental therapeutic studies. This exercise emulates the efforts taken to create the EULAR recommendations for terminology for those at risk of rheumatoid arthritis (RA) ${ }^{14}$. In this Consensus Statement, we describe the process and results of a consensus-building exercise to develop nomenclature for preclinical PsA that can be integrated into future research studies.

\section{Methods \\ Scientific committee}

The consensus exercise was led and designed by the PAMPA study group and the PPACMAN steering committee, composed of methodologists, as well as dermatologists and rheumatologists who are experts in psoriatic disease.

\section{Overview of study design and methods}

In this study, an online Delphi process was used that included international experts in psoriatic disease to achieve consensus on the terminology related to the preclinical phases of PsA for research purposes. The Delphi method is an iterative series of structured rounds that surveys experts until group consensus is reached. This method lends itself well to an online format, which

\footnotetext{
Author addresses

'Department of Dermatology, Harvard Medical School, Brigham and Women's Hospital, Boston, MA, USA.

${ }^{2}$ Department of Medicine, Division of Rheumatology, New York University Langone Health, New York, NY, USA.

${ }^{3}$ Department of Medicine, Division of Rheumatology, University of Toronto, Toronto, ON, Canada.

${ }^{4}$ Psoriatic Arthritis Program, Centre for Prognosis Studies in the Rheumatic Diseases, Krembil Research Institute, University Health Network, Toronto, ON, Canada.

${ }^{5}$ Division of Dermatology, Toronto Western Hospital, University of Toronto, Toronto,

ON, Canada.

${ }^{6}$ Allergy, Immunology, and Rheumatology Division, University of Rochester Medical School, Rochester, NY, USA.

${ }^{7}$ Women's College Research Institute, Women's College Hospital, University of Toronto, Toronto, ON, Canada.

${ }^{8}$ Seattle Rheumatology Associates, Swedish Medical Center and Providence St, Joseph Health, Seattle, WA, USA.

'University of Washington School of Medicine, Seattle, WA, USA.

${ }^{10}$ Department of Medicine, Division of Rheumatology, Center for Clinical Epidemiology and Biostatistics, Perelman School of Medicine, University of Pennsylvania, Philadelphia, PA, USA.

${ }^{11}$ Department of Medicine, Division of Rheumatology, Harvard Medical School, Brigham and Women's Hospital, Boston, MA, USA.

${ }^{12}$ These authors contributed equally: Lourdes M. Perez-Chada, Rebecca H. Haberman.

${ }^{13}$ These authors contributed equally: Joseph F. Merola, Jose U. Scher.
}

enables a larger number of international experts to participate in the survey and avoids any strong influences from a small number of individuals or from standards of practice in certain countries. The Delphi method is used widely in health-care-related research that relies on expert opinion ${ }^{15,16}$; however, some limitations must be acknowledged. The Delphi methodology has been criticized for a possible lack of sufficient details about the background information provided to participants, differing response rates for all rounds, lack of formal feedback between rounds and lack of ability to discuss disagreement directly ${ }^{17,18}$. This Delphi exercise was therefore carefully designed to provide sufficient background detail and feedback for each round and to provide intermittent opportunities for direct discussion. For this study, a pre-Delphi exercise and three rounds of Delphi surveys were used.

Pre-Delphi exercise. Prior to the Delphi development, experts in psoriasis and PsA $(n=28)$ were queried via e-mail regarding terms and definitions of the phases that individuals with psoriasis might go through prior to PsA development. This group of experts was recruited from PPACMAN and also included members of the Group for Research and Assessment of Psoriasis and Psoriatic Arthritis (GRAPPA), an international non-profit organization, and the National Psoriasis Foundation (NPF), a non-profit organization from the USA. On the basis of these results, preliminary terms and definitions were drafted and presented at the PPACMAN 2018 Annual Meeting ${ }^{19}$. After an introductory session, four breakout workshop sessions took place in which attendees suggested changes and provided open-ended opinions about the terms and definitions proposed in the form of small group discussions. These workshop sessions were followed by a plenary session in which the outcomes of each breakout workshop were summarized, and culminated with a voting exercise via an anonymous automated response system. This meeting included dermatologists $(n=8)$, rheumatologists $(n=13)$, rheumatologist-dermatologists $(n=2)$ and industry representatives $(n=17)$. Notably, the industry representatives met in a separate breakout session and did not participate in the voting process to avoid the introduction of bias into the study findings. Further details on the pre-Delphi session can be found in the Supplementary Information. The collected input and results were used to inform the development of the subsequent Delphi survey.

Delphi process. The Delphi survey was designed using feedback from the pre-Delphi exercise. The survey was created and distributed using the New York University REDCap software ${ }^{20}$ to 45 international experts in psoriasis and PsA who were selected by the scientific committee. As with the pre-Delphi exercise, experts were recruited from PPACMAN and included members of GRAPPA and the NPF, and all members who participated in the pre-Delphi exercise were invited to participate in the Delphi exercise. These experts had an average of over 18 years of experience in their fields (TABLE 1). No members of industry were invited to participate. All answers were anonymous, and participants 
Table 1 | Numbers and demographics of participants in each Delphi round

\begin{tabular}{|c|c|c|c|}
\hline Demographic & $\begin{array}{l}\text { First round } \\
(n=29)\end{array}$ & $\begin{array}{l}\text { Second round } \\
(n=33)\end{array}$ & $\begin{array}{l}\text { Third round } \\
(n=35)\end{array}$ \\
\hline \multicolumn{4}{|l|}{ Type of participant } \\
\hline Dermatologist & $5(17.2 \%)$ & $6(18.2 \%)$ & $10(28.6 \%)$ \\
\hline Rheumatologist & $20(69.1 \%)$ & $22(66.7 \%)$ & $22(62.9 \%)$ \\
\hline Rheumatologist-dermatologist & $3(10.3 \%)$ & $3(9.1 \%)$ & $3(8.6 \%)$ \\
\hline Non-clinician researcher & $1(3.4 \%)$ & 2 (6.1\%) & $0(0.0 \%)$ \\
\hline \multicolumn{4}{|l|}{ Location of participant } \\
\hline USA & $18(62.1 \%)$ & $20(60.6 \%)$ & $24(68.6 \%)$ \\
\hline Europe & $7(24.1 \%)$ & $9(27.3 \%)$ & $7(20.0 \%)$ \\
\hline Canada & $4(13.8 \%)$ & $4(12.1 \%)$ & $4(11.4 \%)$ \\
\hline \multicolumn{4}{|l|}{ Gender of participant } \\
\hline Female & $12(41.4 \%)$ & $15(45.5 \%)$ & $16(45.7 \%)$ \\
\hline Male & $17(58.6 \%)$ & $18(54.5 \%)$ & $19(54.3 \%)$ \\
\hline \multicolumn{4}{|c|}{ Amount of experience of participants } \\
\hline Mean number of years (s.d.) & $\begin{array}{l}18.70 \\
(10.47)\end{array}$ & $17.36(10.42)$ & $18.86(11.24)$ \\
\hline \multicolumn{4}{|l|}{ Type of experience of participants } \\
\hline Academic clinicians & $25(86.2 \%)$ & $26(78.8 \%)$ & $28(80.0 \%)$ \\
\hline Clinicians who work privately & $1(3.4 \%)$ & $3(9.1 \%)$ & $3(8.6 \%)$ \\
\hline Both & $3(10.3 \%)$ & $4(12.1 \%)$ & 4 (11.4\%) \\
\hline
\end{tabular}

were asked to vote on and rank their preferred terms and definitions for describing populations of individuals with preclinical PsA. Space for free-form comments was also provided for each question. In all rounds, participants were provided with the results and discussion points generated in the previous rounds, as well as links to published literature to provide background information related to the voting topics ${ }^{3,13}$. Results from the first Delphi round were discussed at the PPACMAN meeting adjacent to the 2019 Annual Meeting of GRAPPA by multiple stakeholders. Consensus for multiple choice and ranking questions was defined a priori as $\geq 70 \%$. For questions that use a visual analogue scale (VAS) ranging from $0 \mathrm{~mm}$ (should not be considered) to $100 \mathrm{~mm}$ (should definitely be considered), items were retained if the median score was $>70 \mathrm{~mm}$. If consensus was not reached, the question was carried through to the next round; however, Delphi items rated on a VAS that had a median score of $<60 \mathrm{~mm}$ were removed.

Data analysis. Descriptive statistics were used to report the voting results. Continuous data are presented as medians and interquartile range unless otherwise noted.

\section{Results}

\section{Pre-Delphi exercise}

At the 2018 PPACMAN Annual Meeting, stakeholders were presented with preliminary terms and definitions that were informed by the input collected from the initial survey distributed by e-mail. Expanded discussions and voting followed, but no consensus was reached. Further details of the pre-Delphi exercise are provided in the Supplementary Information.

\section{Delphi exercise}

Round 1 of the Delphi exercise received 29 responses (response rate $64.4 \%$ ), round 2 received 33 responses (response rate $73.3 \%$ ) and round 3 received 35 responses (response rate $77.7 \%$ ). Although the invited participants varied with regard to demographics and experience, the respondents were rheumatologists, dermatologists and rheumatologist-dermatologists who were mostly academic clinicians and/or researchers (TABLE 1). The original terms and definitions presented for voting in the Delphi exercise are provided in the Supplementary Information. BOX 1 shows the final terms and definitions proposed after consensus was reached.

\section{Terms and definitions}

Individuals with psoriasis at increased risk for PsA. During round 1 of the Delphi exercise, $80 \%$ of the panellists agreed on the term 'increased risk for PsA', and 86\% voted that this term defines a meaningful subgroup for future research studies. Other terms proposed included 'at risk', 'high risk,' 'higher risk' and 'elevated risk. The term 'at risk' for PsA was not favoured by participants, who noted that any patient with psoriasis has the potential to develop PsA. During round 1, consensus for the definition was not reached.

In round 2 of the Delphi exercise, the definition of 'any individual with psoriasis and one or more risk factors for progression to PsA' reached consensus with $84.4 \%$ agreement. The alternative definition proposed was 'any individual with psoriasis and one or more risk factors for progression to synovio-entheseal disease'. Participants noted that the term synovio-entheseal disease might not be commonly used, particularly by dermatologists.

Regarding which risk factors for progression from psoriasis to PsA should be considered, obesity, the presence of arthralgia, severe psoriasis, a history of uveitis, nail psoriasis, scalp psoriasis and having a first-degree relative with PsA reached consensus in round 1 of the Delphi exercise (median $>70 \mathrm{~mm}$ on a $100-\mathrm{mm}$ VAS), whereas any associated gene (such as $H L A-B^{*} 08$, $H L A-B^{\star} 27, H L A-B^{\star} 38$ or $\left.H L A-B^{\star} 39\right)$ reached consensus in round 2 of the Delphi exercise (Supplementary Figure 1).

Individuals with psoriasis and asymptomatic synovioentheseal imaging abnormalities. In round 1 of the Delphi exercise, the terms 'subclinical PsA', 'potential PsA', 'psoriasis with imaging findings', 'psoriasis with asymptomatic synovio-entheseal imaging findings' and 'psoriasis with imaging abnormalities' were proposed (Supplementary Table 1). Subclinical PsA was generally disliked because the term implies that patients will definitely go on to develop PsA. As consensus was not reached, the three terms with the highest number of votes were moved to round 2 of the Delphi exercise. In round 2 , the term 'psoriasis with asymptomatic synovioentheseal imaging abnormalities' outstripped the other terms, gaining almost $60 \%$ of the votes. Consensus was finally reached for this term by $85.7 \%$ of participants in round 3 of the Delphi exercise. Overall, $86.2 \%$ of the 


\section{Box 1 | PAMPA consensus terminology for preclinical phases of PsA}

According to the proposed terminology, in prospective research studies, individuals would be described in the following ways:

\section{Individuals with psoriasis at increased risk for PsA}

Any individual with psoriasis and one or more risk factors for progression to psoriatic arthritis (PsA).

- Risk factors include obesity, the presence of arthralgia, severe psoriasis, a history of uveitis, nail psoriasis, scalp psoriasis, having a first-degree relative with PsA and any associated gene (such as HLA-B*08, HLA-B*27, HLA-B*38 or HLA-B*39).

- Can be combined with either of the other two terms.

Individuals with psoriasis and asymptomatic synovio-entheseal imaging abnormalities

Any individual with psoriasis and imaging evidence of synovio-entheseal abnormalities that is not associated with clinical signs or symptoms.

- Imaging modalities include MRI for axial disease, MRI for peripheral arthritis, ultrasonography for peripheral arthritis, ultrasonography for enthesitis and plain radiography for peripheral arthritis. Specific MRI findings used to define imaging abnormalities include enthesitis, bone marrow oedema, synovitis, tendonitis, bone erosions and new bone formation. Specific ultrasonography findings used to define imaging abnormalities include enthesitis, synovitis, tendonitis and bone erosions.

- Can be combined with 'individuals with psoriasis at increased risk for PsA'; for example, an individual with psoriasis might have uveitis (a risk factor for PsA) and have asymptomatic enthesitis defined by ultrasonography.

- Cannot be combined with 'individuals with psoriasis and musculoskeletal symptoms not explained by other diagnosis'.

Individuals with psoriasis and musculoskeletal symptoms not explained by other diagnosis

Any individual with psoriasis and heel pain, stiffness and/or arthralgia not explained by another diagnosis.

- Can be combined with 'individuals with psoriasis at increased risk for PsA'; for example, an individual with psoriasis might have uveitis (a risk factor for PsA) and have heel pain that is not explained by another diagnosis.

- Cannot be combined with 'individuals with psoriasis and asymptomatic synovioentheseal imaging abnormalities'. formation $(80 \mathrm{~mm})$ (Supplementary Figure 3). For ultrasonography, enthesitis (median on a $100-\mathrm{mm}$ VAS $=89 \mathrm{~mm})$, synovitis $(97 \mathrm{~mm})$, tendonitis $(77 \mathrm{~mm})$ and bone erosions $(87 \mathrm{~mm})$ all met consensus in round 1 of the Delphi exercise (Supplementary Figure 4).

Individuals with psoriasis and musculoskeletal symptoms not explained by other diagnosis. During round 1 of the Delphi exercise, $93.1 \%$ of participants agreed on the term 'psoriasis with musculoskeletal symptoms not explained by other diagnosis' (BOX 1). In round 2 of the Delphi exercise, $87.9 \%$ voted that this term was meaningful for future research studies. Previous iterations of this term included 'prodromal PsA', 'psoriasis with arthralgia,' 'psoriasis with musculoskeletal symptoms' and 'psoriasis with musculoskeletal symptoms without musculoskeletal signs'. Similar to the term 'subclinical PsA', participants argued that the term 'prodromal PsA' implied that all patients with psoriasis would progress to PsA and was therefore inappropriate.

To define which musculoskeletal symptoms should be considered, participants were provided with a list of symptoms that had previously been identified in the literature as predictors of PsA, along with their hazard ratios and $95 \%$ confidence intervals ${ }^{21}$. Participants then scored the factors on a VAS, and a median score of $\geq 70 \mathrm{~mm}$ was defined as reaching consensus. Of these factors, heel pain, stiffness and arthralgia all reached consensus (median scores of $84 \mathrm{~mm}, 80.5 \mathrm{~mm}$ and $75 \mathrm{~mm}$, respectively), whereas fatigue and problems with physical function (median scores of $67.5 \mathrm{~mm}$ and $51 \mathrm{~mm}$, respectively) did not.

Terms and definitions that did not achieve consensus. Participants were also asked to comment on time points after the diagnosis of PsA, specifically looking at the 6-month and 24-month time points. Although consensus was reached for 6 months from diagnosis being a meaningful time point (90.9\%), consensus was not reached on whether this population of individuals should be termed 'early PsA', 'very early PsA' or 'new onset PsA'. Similarly, consensus was not reached on whether diagnosis should be defined by satisfying Classification for Psoriatic Arthritis (CASPAR) criteria ${ }^{22}$ and/or musculoskeletal symptom onset. There was a lack of consensus that 24 months was a meaningful time point. psoriasis and imaging evidence of synovio-entheseal abnormalities that is not associated with clinical signs or symptoms' ( $88.6 \%$ of the votes).

Imaging modalities that reached consensus for use to define 'imaging abnormalities' in round 1 of the Delphi exercise included MRI for axial disease (median on a $100-\mathrm{mm}$ VAS $=97 \mathrm{~mm}$ ), MRI for peripheral arthritis $(86 \mathrm{~mm})$, ultrasonography for peripheral arthritis (90 $\mathrm{mm})$, ultrasonography for enthesitis $(90 \mathrm{~mm})$ and plain radiography for peripheral arthritis $(70 \mathrm{~mm})$ (Supplementary Figure 2). Participants also voted on which specific MRI and ultrasonography signs should be considered for use in defining imaging abnormalities. MRI signs that met consensus for inclusion were enthesitis (median on a 100-mm VAS $=90 \mathrm{~mm}$ ), bone marrow oedema $(90 \mathrm{~mm})$, synovitis $(95 \mathrm{~mm})$, tendonitis $(70 \mathrm{~mm})$, bone erosions $(81 \mathrm{~mm})$ and new bone

\section{Future research agenda}

During the face-to-face PPACMAN meeting adjacent to the 2019 Annual Meeting of GRAPPA, the results of the consensus exercise were presented, and several areas were identified as priorities for investigation.

\section{Imaging}

Imaging studies (primarily ultrasonography or MRI modalities) have the potential to improve the definition of meaningful subclinical inflammatory states and their ability to predict PsA development. In particular, ultrasonography represents a feasible and adaptable modality that is already being applied in the clinical setting to identify patients with psoriasis who have subclinical enthesitis and/or synovitis ${ }^{23-25}$. High-resolution peripheral 
quantitative computed tomography (HR-pQCT) also shows promise for use in assessing if the presence of structural entheseal lesions can predict future PsA among patients with psoriasis ${ }^{26}$. Whether sonographic findings (such as synovitis, enthesitis, tenosynovitis or peritonitis) or radiological findings (such as bone erosions, tenosynovitis or bone proliferations) represent abnormal inflammatory features or are simply physiological immune-mediated responses aimed at containing disease progression remains a subject of intense debate. However, reports that the treatment of psoriasis using IL-12-IL-23 blockade ${ }^{27,28}$ or IL-17 blockade ${ }^{29}$ in patients without overt joint symptoms resulted in suppression of these sonographic and radiological abnormalities are promising and will surely aid in the design of future preventive studies, particularly if paired with clinical and molecular risk factor enrichment strategies. These studies will likewise inform a future revision of the term 'psoriasis with musculoskeletal symptoms not explained by other diagnosis'.

\section{Prodromal phase and non-specific pain}

In an attempt to characterize a 'preclinical' phase of PsA, the term 'psoriasis with musculoskeletal symptoms not explained by other diagnosis' was selected by stakeholders over 'prodromal' and 'preclinical', as these terms might imply that the progression from psoriasis to PsA is definite. Indeed, the terms 'preclinical' or 'prodromal' PsA can only be applied retrospectively at this time owing to a lack of ability to truly predict progression to PsA. Currently, limited data exist with which to conclusively define the non-specific musculoskeletal symptoms that should be considered as part of this phase for research purposes ${ }^{21}$. Further understanding of this stage is crucial to distinguish who might be at the highest risk of progression to PsA and, ultimately, to implement early treatment and prevention strategies for PsA. Information gathered from ongoing ${ }^{30}$ and future longitudinal studies of musculoskeletal symptoms will be needed to further inform and revise this definition.

\section{Preventive trial design}

A preventive medicine approach is not foreign to the field of rheumatology and chronic immune-mediated inflammatory diseases. Specifically, investigators have pioneered trials in preclinical or pre-damage stages of systemic lupus erythematosus and RA, resulting, in some cases, in improved outcomes and even prevention ${ }^{31-35}$. Several prevention trials supported by the US National Institutes of Health are currently underway, including the SMILE study ${ }^{35}$ and the StopRA study ${ }^{36}$, and many other studies are in progress in Europe ${ }^{37}$.

Although the field of psoriatic disease is rapidly moving forward in the design of preventive trials, several questions inevitably remain unanswered and will require a retrospective analytical understanding of the psoriasis-to-PsA transition to be achieved before they can be answered and preventive trials can be initiated. Chief among those questions is how to ascertain the relative weight for each proposed risk factor (in other words, the risk enrichment). The clinical, demographic, genetic and molecular features currently associated with progression from psoriasis to PsA have been mostly derived from retrospective and cross-sectional studies. One strategy for identifying relevant risk factors for progression in prospective studies consists of studying individuals with psoriasis at increased risk of developing PsA who have at least moderate skin disease and imaging evidence of entheseal abnormalities, plus one or more of the following features: scalp involvement, psoriatic nail disease or genetic factors linked to progression (such as a first-degree relative with PsA) $)^{38}$.

The specific therapeutic approach in prevention trials will also be challenging, as arguments exist for using medications with any of the available mechanisms of action (such as TNF inhibitors, IL-17 inhibitors, IL-23 inhibitors or phosphodiesterase 4 inhibitors). Importantly, the role of natural history registries (following patients on immunomodulatory therapies as well as those with psoriasis who elect not to be treated with systemic medications) will be of the utmost relevance, as the ultimate goal will be to create a risk-score that incorporates the relative predictive value of each of the proposed risk factors alongside rigorous cut-off thresholds for sensitivity and specificity.

\section{Conclusions}

Given that psoriasis commonly precedes the development of $\mathrm{PsA}^{39}$, a unique pre-disease window of opportunity exists in the psoriasis-to-PsA continuum for studies on the clinical and molecular features of transition. To capitalize on this window of opportunity, it is imperative that the preclinical stages of PsA are better understood. The terms and definitions developed by the PAMPA study group describe clinical and imaging features of pre-disease states that individuals might traverse prior to PsA development. The use of standardized nomenclature and common definitions for PsA research will help to facilitate communication and comparison across future studies, which will enable robust validation between efforts in this particularly complex and heterogeneous disease. Overall, it is hoped that the consensus definitions set out in this Consensus Statement will catalyse the development of preventive strategies and, ultimately, improve outcomes in $\mathrm{PsA}^{13}$.

Importantly, the overarching output derived from this consensus exercise is to be used exclusively for research purposes at this time. Although necessarily an evolving process, this work represents a much-needed starting point. Furthermore, this terminology should not be viewed as restrictive or unchangeable, nor should it be used for clinical care, given the preliminary nature of these terms and definitions. Adoption of terminology in the clinical sphere will require a natural refinement process and an iterative validation approach as research in this area progresses. Together, these efforts will characterize novel clinical and molecular features associated with the transition of psoriasis to PsA, which in turn will oblige the field to revise the proposed definitions and risk factors.

Published online 15 February 2021 
1. Mease, P. J. et al. Prevalence of rheumatologistdiagnosed psoriatic arthritis in patients with psoriasis in European/North American dermatology clinics. J. Am. Acad. Dermatol. 69, 729-735 (2013)

2. Alinaghi, F. et al. Prevalence of psoriatic arthritis in patients with psoriasis: a systematic review and meta-analysis of observational and clinical studies. J. Am. Acad. Dermatol. 80, 251-265.e219 (2019).

3. Eder, L. et al. The incidence and risk factors for psoriatic arthritis in patients with psoriasis: a prospective cohort study. Arthritis Rheumatol. 68 915-923 (2016)

4. Lee, S., Mendelsohn, A. \& Sarnes, E. The burden of psoriatic arthritis: a literature review from a global health systems perspective. P T 35, 680-689 (2010).

5. Husted, J. A., Gladman, D. D., Farewell, V. T. \& Cook, R. J. Health-related quality of life of patients with psoriatic arthritis: a comparison with patients with rheumatoid arthritis. Arthritis Rheum. 45, 151-158 (2001)

6. Kimball, A. B., Jacobson, C., Weiss, S., Vreeland, M. G. $\& W u, Y$. The psychosocial burden of psoriasis. Am. J. Clin. Dermatol. 6, 383-392 (2005).

7. Tillett, W. et al. Factors influencing work disability in psoriatic arthritis: first results from a large UK multicentre study. Rheumatology 54, 157-162 (2015).

8. Armstrong A. W., Robertson, A. D., Wu, J., Schupp, C. \& Lebwohl, M. G. Undertreatment, treatment trends, and treatment dissatisfaction among patients with psoriasis and psoriatic arthritis in the United States: findings from the National Psoriasis Foundation surveys, 2003-2011. JAMA Dermatol. 149, 1180-1185 (2013).

9. Villani, A. P. et al. Prevalence of undiagnosed psoriatic arthritis among psoriasis patients: systematic review and meta-analysis. J. Am. Acad. Dermatol. 73, 242-248 (2015)

10. Gladman, D. D., Thavaneswaran, A., Chandran, V. \& Cook, R. J. Do patients with psoriatic arthritis who present early fare better than those presenting later in the disease? Ann. Rheum. Dis. 70, 2152-2154 (2011)

11. Haroon, M. Gallagher, P. \& FitzGerald, O. Diagnostic delay of more than 6 months contributes to poor radiographic and functional outcome in psoriatic arthritis. Ann. Rheum. Dis. 74, 1045-1050 (2015).

12. Haberman, R. et al. Bridging the gaps in the care of psoriasis and psoriatic arthritis: the role of combined clinics. Curr. Rheumatol. Rep. 20, 76 (2018).

13. Scher, J. U., Ogdie, A., Merola, J. F. \& Ritchlin, C. Preventing psoriatic arthritis: focusing on patients with psoriasis at increased risk of transition. Nat. Rev. Rheumatol. 15, 153-166 (2019).

14. Gerlag, D. M. et al. EULAR recommendations for terminology and research in individuals at risk of rheumatoid arthritis: report from the Study Group for risk factors for rheumatoid arthritis. Ann. Rheum. Dis. 71, 638-641 (2012).

15. Burt, C. G. et al. Developing a research agenda for the American Society of Colon and Rectal Surgeons: results of a Delphi approach. Dis. Colon Rectum $\mathbf{5 2}$, 898-905 (2009).

16. Steurer, J. The Delphi method: an efficient procedure to generate knowledge. Skeletal Radiol. 40, 959-961 (2011).

17. Waggoner, J., Carline, J. D. \& Durning, S. J. Is there a consensus on consensus methodology? Descriptions and recommendations for future consensus research. Acad. Med. 91, 663-668 (2016)

18. Humphrey-Murto, S. et al. Consensus building in OMERACT: recommendations for use of the Delphi for core outcome set development. J. Rheumatol. 46 , 1041-1046 (2019).

19. Haberman, R. et al. Psoriasis and psoriatic arthritis clinics multicenter advancement network consortium (PPACMAN) 2018 Annual Meeting summary. J. Psoriasis Psoriatic Arthritis 5, 68-72 (2020).

20. Harris, P. A. et al. Research electronic data capture (REDCap) - a metadata-driven methodology and workflow process for providing translational research informatics support. J. Biomed. Inform. 42, 377-381 (2009).

21. Eder, L. et al. The development of psoriatic arthritis in patients with psoriasis is preceded by a period of nonspecific musculoskeletal symptoms: a prospective cohort study. Arthritis Rheumatol. 69, 622-629 (2017).

22. Taylor, W. et al. Classification criteria for psoriatic arthritis: development of new criteria from a large international study. Arthritis Rheum. 54, 2665-2673 (2006).

23. Eder, L., Barzilai, M., Peled, N., Gladman, D. D. \& Zisman, D. The use of ultrasound for the assessment of enthesitis in patients with spondyloarthritis. Clin. Radiol. 68, 219-223 (2013).

24. D'Agostino, M. A. Ultrasound imaging in spondyloarthropathies. Best Pract. Res. Clin. Rheumatol. 24, 693-700 (2010).

25. Elalouf, O. et al. Psoriatic arthritis sonographic enthesitis instruments: a systematic review of the literature. J. Rheumatol. 46, 43-56 (2019).

26. Simon, D. et al. Structural entheseal lesions in patients with psoriasis are associated with an increased risk of progression to psoriatic arthritis. Arthritis Rheumatol. https://doi.org/10.1002/art.41239 (2020).

27. Savage, L. et al. Regression of peripheral subclinical enthesopathy in therapy-naive patients treated with ustekinumab for moderate-to-severe chronic plaque psoriasis: a fifty-two-week, prospective, open-label feasibility study. Arthritis Rheumatol. 71, 626-631 (2019).

28. Araujo, E. G. et al. Effects of ustekinumab versus tumor necrosis factor inhibition on enthesitis: results from the enthesial clearance in psoriatic arthritis (ECLIPSA) study. Semin. Arthritis Rheum. 48 632-637 (2019)

29. Kampylafka, E. et al. Disease interception with interleukin-17 inhibition in high-risk psoriasis patients with subclinical joint inflammation-data from the prospective IVEPSA study. Arthritis Res. Ther. $\mathbf{2 1}$ 178 (2019).

30. Eder, L. et al. Health care utilization for musculoskeleta issues during the pre-diagnosis period in psoriatic arthritis - a population-based study. Arthritis Care Res. https://doi.org/10.1002/acr.24146 (2020).

31. Burmester, G. R. et al. Safety and efficacy of upadacitinib in patients with rheumatoid arthritis and inadequate response to conventional synthetic disease-modifying anti-rheumatic drugs (SELECT-NEXT) a randomised, double-blind, placebo-controlled phase 3 trial. Lancet 391, 2503-2512 (2018).

32. Deane, K. D., Striebich, C. C. \& Holers, V. M. Prevention of rheumatoid arthritis: now is the time, but how to proceed? Arthritis Rheumatol. 69, 873-877 (2017).

33. Gerlag, D. M. et al. Effects of B-cell directed therapy on the preclinical stage of rheumatoid arthritis: the PRAIR study. Ann. Rheum. Dis. 78, 179-185 (2019).

34. Al-Laith, M. et al. Arthritis prevention in the preclinical phase of RA with abatacept (the APIPPRA study): a multi-centre, randomised, double-blind, parallel-group, placebo-controlled clinical trial protocol. Trials 20, 429 (2019).

35. Olsen, N. J. et al. Study of anti-malarials in incomplete lupus erythematosus (SMILE): study protocol for a randomized controlled trial. Trials 19, 694 (2018)

36. US National Library of Medicine. ClinicalTrials. gov https://clinicaltrials.gov/ct2/show/study/NCT02603146 (2021).

37. Mahler, M., Martinez-Prat, L., Sparks, J. A. \& Deane, K. D. Precision medicine in the care of rheumatoid arthritis: focus on prediction and prevention of future clinically-apparent disease. Autoimmun. Rev. 19, 102506 (2020).

38. Ritchlin, C. \& Scher, J. U. Strategies to improve outcomes in psoriatic arthritis. Curr. Rheumatol. Rep. 21, 72 (2019)

39. Gladman, D. D., Antoni, C., Mease, P., Clegg, D. O. $\&$ Nash, P. Psoriatic arthritis: epidemiology, clinical features, course, and outcome. Ann. Rheum. Dis. 64 ii 14-17 (2005).

\section{Acknowledgements}

We thank all stakeholders who participated in the Delphi study and consensus meetings. The work of the authors is supported by grants from the US National Institutes of Health and the National Institute of Arthritis and Musculoskeletal and Skin Diseases (R01AR074500-01A to J.U.S. and T32AR069515 to R.H.H.) and by The Riley Family Foundation and The Snyder Family Foundation (J.U.S.).

\section{Author contributions}

L.M.P.-C., R.H.H., J.F.M. and J.U.S. researched data for the article. L.M.P.-C., R.H.H., S.R., A.O., J.F.M. and J.U.S. provided substantial contributions to discussions of the content and wrote the article. All authors reviewed and/or edited the manuscript before submission.

\section{Competing interests}

R.H.H. declares they have received consultation honoraria from Janssen. V.C. declares they have received research grants and/or advisory board honoraria from AbbVie, Amgen, BMS, Celgene, Eli Lilly, Janssen, Novartis, Pfizer and UCB. V.C. also declares that their spouse is employed by Eli Lilly. C.F.R. declares they have received consultation and/or investigator honoraria from AbbVie, Eli Lilly and Novartis. L.E. declares they have received consultation honoraria and non-restricted research and educational grants from AbbVie, Amgen, Eli Lilly, Janssen, Novartis, Pfizer and UCB. P.M. declares they have received research grants, consultation and/or speaker honoraria from AbbVie, Amgen, BMS, Boehringer Ingelheim, Celgene, Eli Lilly, Galapagos, Gilead, GlaxoSmithKline, Janssen, Novartis, Pfizer, Sun Pharma and UCB. S.R. declares they have received consultation honoraria from Amgen, Janssen, Novartis and Pfizer and have been involved in clinical trials with Amgen and Celgene. A.O. declares they have received consultation honoraria from AbbVie, Amgen, BMS, Celgene, Corrona, Eli Lilly, Janssen, Novartis and Pfizer and have received grant support from Amgen (to Forward/National Databank for Rheumatic Disease) and from Novartis and Pfizer (to the University of Pennsylvania). J.F.M. declares they have received consultation and/or investigator honoraria from AbbVie, Arena, Avotres, Biogen, Celgene, Dermavant, Eli Lilly, EMD Sorono, Janssen, Leo Pharma, Merck, Novartis, Pfizer, Regeneron, Sanofi, Sun Pharma and UCB. J.U.S. declares they have received consultation and/or investigator honoraria from AbbVie, Janssen, Novartis, Pfizer, Sanofi and UCB. All other authors declare no competing interests.

\section{Peer review information}

Nature Reviews Rheumatology thanks A. Hueber, E. Lubrano, $\mathrm{Y}$.-Y. Leung and the other, anonymous, reviewer(s) for their contribution to the peer review of this work.

\section{Publisher's note}

Springer Nature remains neutral with regard to jurisdictional claims in published maps and institutional affiliations.

\section{Supplementary information}

The online version contains supplementary material available at https://doi.org/10.1038/s41584-021-00578-2.

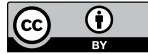

Open Access This article is licensed under a Creative Commons Attribution 4.0 International License, which permits use, sharing, adaptation, distribution and reproduction in any medium or format, as long as you give appropriate credit to the original author(s) and the source, provide a link to the Creative Commons license, and indicate if changes were made. The images or other third party material in this article are included in the article's Creative Commons license, unless indicated otherwise in a credit line to the material. If material is not included in the article's Creative Commons license and your intended use is not permitted by statutory regulation or exceeds the permitted use, you will need to obtain permission directly from the copyright holder. To view a copy of this license, visit http://creativecommons.org/licenses/ by $/ 4.0 \%$.

(c) The Author(s) 2021 